\title{
The relevance of situation awareness in older adults' cognitive functioning: a review
}

\author{
Ryan J. Caserta $\cdot$ Lise Abrams
}

Received: 6 July 2006 / Accepted: 13 February 2007 / Published online: 13 March 2007

(C) EGREPA 2007

\begin{abstract}
Age-related declines in cognition may have detrimental effects on older adults' ability to complete everyday activities that young- and middle-aged individuals perform automatically. Theories of cognitive aging have found deficits in older adults' fluid intelligence, capacity for inhibition, number of processing resources, and speed of processing, and in recent years, studies have proposed cognitive strategies to ameliorate these declines. However, few strategies directly train the cognitive strategies necessary to improve performance in dynamic environments and physical activities. One such strategy may be the enhancement of situation awareness, the capability to perceive and understand one's environment. Although the term has typically been applied to pilots and other expert performers, situation awareness may also be relevant to cognitive aging, where older adults' perception and comprehension of their environment become critical to everyday functioning and physical activities. If older adults' situation awareness can be facilitated, then it may be possible to reduce the impact of age-related cognitive declines, allowing older adults to successfully participate in dynamic situations and sports where the environment is constantly changing (e.g., driving
\end{abstract}

Portions of this paper were reported at the annual meeting of the North American Society for the Psychology of Sport and Physical Activity in June, 2005.

\footnotetext{
L. Abrams $(\bowtie)$

Department of Psychology, University of Florida,

P.O. Box 112250, Gainesville, FL 32611-2250, USA

e-mail: abrams@ufl.edu

\section{R. J. Caserta}

Department of Applied Physiology and Kinesiology,

University of Florida,

P.O. Box 11825, 100 Florida Gym,

Gainesville, FL 32611-8205, USA

e-mail: rcaserta@ufl.edu
}

and tennis). The following review outlines cognitive deficits in aging, details their relation to situation awareness, and discusses how training in situation awareness may reduce cognitive declines.

Keywords Situation awareness · Cognitive aging · Conceptual representation · Training · Physical activity

Over the past four decades, research examining age-related differences in cognition has increased significantly, prompting Salthouse [54] to propose a need for theoretical frameworks to organize and integrate these research findings as well as determine the direction of future research. Along with an increase in theoretical development, cognitive aging research has moved beyond the documentation of age-related deficits (e.g., [26, 27, 41, 55]) to examine how physical activity and cognitive training can improve cognitive functioning [1, 3, 11, 31, 37, 49]. For scientific theories of cognitive aging to be practical for older adults, research must address the development and effective distribution of beneficial techniques that slow or eliminate age-related deficits in cognitive functioning, especially those relating to dynamic environments.

Specifically, the improvement of situation awareness may potentially have a significant impact on reducing agerelated declines. Situation awareness has been generally defined as "the perception of elements in the environment within a volume of time and space, the comprehension of their meaning, and the projection of their status in the near future" ([15], p. 97). Simply stated, situation awareness refers to the ability to know what is going on around you. Situation awareness provides a keen understanding of the environment, especially during unfamiliar, dynamic situations. In the military and sport psychology literatures, situation awareness has been used to measure the percep- 
tual levels of performers and to influence decision-making capabilities but, more importantly, to understand how such behaviors of interest can be improved across a variety of performers in varied environments/sports. Therefore, the purpose of this review is to bring situation awareness into the aging literature by detailing its relationship to known age-related deficits in cognitive processing with the hope of encouraging future research on intervention strategies.

Situation awareness begins with conceptual representation, which is vital to cognitive operations and is intricately tied to memory, perception, decision making, actions, and inductive inferences [25]. A concept is constructed from an infinite set of representations from the environment. The conceptual system organizes a collection of categorical images representing individual components of the experience rather than full-length recreations [5]. Although selectively attending to changes in the environment, the conceptual system categorizes information into components, which are then stored in memory [58]. The ability to store countless components in memory for use in future interactions lies in the conceptual system's ability to support perception of the situation. However, the conceptual system goes beyond representation of experienced events by utilizing relevant components during unfamiliar, atypical situations. Conceptual representations become vital when heightened awareness of situations is required, because the mental prototype formed from the human conceptual system provides information used in all aspects of situation awareness.

Situation awareness has been described as the complete understanding of factors that will contribute to the optimal performance of a task under expected and unexpected conditions [51]. Externally paced situations, which are temporally driven, require heightened visual attention to preliminary movements and cues. Selectively attending to relevant advanced cues allows individuals to make anticipated decisions as to intentions, which increases the speed of reactions. Quick responses become less important when driving a car on a desolate highway; however, vast differences exist when driving in downtown New York City during rush hour. Attempting to simultaneously attend to or become consumed by all the traffic signs, traffic lights, cars, bike messengers, and pedestrians would be ineffective and dangerous, especially when quick decisions must be made. Equally ineffective and dangerous would be narrowing one's attentional field to the point of becoming consumed by only one singular object, such as the car directly in front of you. For situation awareness to be effective, situational assessments must actively access coherent conceptual representations, as each experience expands an individual's current knowledge base while influencing the acquisition and interpretation of new knowledge.

Although situation awareness and conceptual representation are terms not typically discussed within the context of cognitive aging, they seem relevant to the potential dangers and difficulties that older adults face when completing everyday tasks (e.g., crossing a busy street, driving on familiar and unfamiliar roads, cooking, reaching for and/or storing items in the home, etc.). Consider the task of crossing the street in a busy metropolitan city. In terms of an appropriate conceptual representation, the concept street is different depending on the location and time of day. A neighborhood street may be familiar, but many aspects of the scene are constantly changing, such as the amount and speed of traffic or are atypical (e.g., imperfections in the street or sidewalk, wet pavement from bad weather conditions, and driver error such as illegally turning right on red). Older adults are at a disadvantage in these situations because of the dynamic environment, which requires frequent updating of conceptual representations - a task made more difficult by age-related declines in cognitive functioning (e.g., reduced attention and working memory capacity and slowed processing speeds $[12,36,39,40,57,60])$. Heightened awareness of situations may facilitate the construction of appropriate conceptual representations, minimizing older adults' susceptibility to falls or getting struck by a vehicle when crossing a busy street. The enhancement of situation awareness and appropriate conceptual representation becomes increasingly more important to older adults when considering the long-term damage and mortality that occurs from falls [24, 59]. Recent findings indicate that $96 \%$ of home dwelling individuals with dementia suffer from falling incidences, causing fractures or dislocations $60.8 \%$ of the time [52]. Even healthy adults suffer from falling incidents; however, the development of exercise programs have been found to decrease falls by $46 \%$ and increase obstacle avoidance by $12 \%$ [61]. Although improving physical attributes (i.e., strength, balance, and coordination) has been one approach to reducing falls, developing perceptual-cognitive training programs that enhance older adults' perceptual abilities may be an additional method for increasing those percentages.

\section{Levels and measurement of situation awareness}

\section{Level 1 situation awareness}

Researchers examining individuals where situation awareness is essential in peak performance (e.g., pilots, air traffic controllers, medical doctors) have determined the importance of the three levels of situation awareness [15]. Level 1 consists of the perception of essential cues. The perception of cues or vital information is necessary when creating an accurate picture of the situation. More specifically, individuals are visually determining what cues are present, the location of these cues, as well as the speed in which the cues are moving (i.e., temporal-spatial aspects). When 
testing expert decision makers, empirical evidence has found that pattern recognition was used in the perception of cues $[19,33]$. Problems in perception have been traced to failures or deficiencies in the mechanical system or problems with cognitive processes. The lack of this fundamental perceptual ability has been found to produce $76 \%$ of situation awareness errors in pilot performance [30]. In this situation, a Level 1 situation awareness error may consist of failing to recognize an airplane on the runway during landing or identifying other airplanes that are close to intersecting the intended heading.

\section{Level 2 situation awareness}

Level 2 deals with comprehension, which encompasses how individuals combine, interpret, store, and retain information. Performing in dynamic, time-demanding situations requires rapid integration of multiple cues while deciphering between the cues that are relevant and irrelevant to the outcome of the task. The meaning or diagnoses of relevant cues are made, and patterns are detected. Comprehension can also be defined with regards to alternatives [45]. If one of the primary purposes of situation awareness is to improve decision making, increased comprehension of pertinent cues allows individuals to make accurate decisions from an infinite number of alternative responses. Level 2 situation awareness can also be considered as a state or process that has been linked to attention management [44]. The emphasis is placed on how to represent context, not to limit it. In addition, the detection of leverage points becomes an important aspect of Level 2. Leverage points are temporally and spatially defined within dynamic environments and are a key function in expertise [35]. For example, for a fighter pilot, the leverage point is the opportunity to seize control of a dogfight with a perfectly timed tactical maneuver. Jones and Endsley [30] found that $20 \%$ of the situation awareness errors occurred at the comprehension level (Level 2). These errors are less about recognition and more about lack of understanding. Although a pilot may perceive a strong crosswind during landing, the error occurs in not understanding how this affects his/her approach.

\section{Level 3 situation awareness}

Finally, Level 3 situation awareness, the highest level of understanding, consists of the propensity to predict future situation events and dynamics. The ability to project from past knowledge to current and future situations impacts the temporal-spatial aspects of the performance, including the timing of situation-specific actions and the decisions that are made under duress. Projection can also occur backward into the past, which has been explained through the process of mental simulations [34]. By creating stories, predictions are made about the future from explanations of the past. Although future events and outcomes remain highly unpredictable, expert performers who tap into this third level of situation awareness have a distinct advantage over the competition. Ultimately, Level 3 is where elite level performers in any domain are distinguished from others. Pilots who have attained Level 3 situation awareness are capable of avoiding such errors described in Levels 1 and 2 and also have the capacity to handle other concerns while being prepared for potential emergencies.

As described above, the use of all three levels of situation awareness has primarily been confined to the field of aviation. However, further investigations have been conducted in the fields of air traffic control and medicine. For air traffic controllers, situation awareness has been used to examine and decrease the number of air traffic control incidents. The use of situation awareness has included the following assessments: knowledge of aircraft location, aircraft callsign/altitude/groundspeed/heading/next sector/ type/activity, determination of the aircraft that has been issued incomplete assignments and correct assignments, determination of the aircrafts that are conforming to their assignments, determination of aircrafts that are experiencing an emergency, and determination of aircrafts that are violating minimum requirements, to name a few (see [48] for a review). In the field of medicine, specifically anesthesiology, situation awareness has become a key component in providing optimal patient care. Situation awareness has been used to improve the ability of anesthesiologists to detect, diagnose, and make the correct decisions when treating critical incidents. Medical simulators and analysis of real cases are being used to investigate and train future anesthesiologists (see [13, 23] for a review).

Effective situational analysis should increase the probability of anticipating dynamic events. In turn, such appropriate situation awareness behaviors should enhance the probability of making better decisions/actions in such situations. Therefore, individuals performing in such changing environments must develop the appropriate perception of fundamental cues (Level 1) then expand on those context-relevant perceptions through the comprehension of what those cues mean in accordance with the current situation (Level 2) and ultimately apply this newly acquired understanding to future situations (Level 3). The importance of situation awareness in each of its three levels is best understood when considering the demands of driving in heavy traffic. Information-rich cues include the speed in which vehicles are traveling, traffic signs and signals, and seeing beyond the vehicle in front of you to other vehicles, indicators, lights, and signs. Knowing that these are the most important cues is not enough. Compre- 
hending the information being provided from these cues is the key. For example, attending to the brake lights of the vehicles that are two or more cars ahead of you can allow you to anticipate traffic coming to a stop. Comprehending such cues and learning from these past experiences may then affect reacting to similar driving situations in the future as such experiences enhance Level 3.

As shown in the distinguishing characteristics of each level of situation awareness, a combination of pattern recognition, conscious analysis, story building, mental simulation, and metacognitive processes are all used at various times to form situation awareness [18]. Because of these cognitive components, age-related declines may occur across each of the three levels of situation awareness. However, little research to date has directly examined the effects of aging on situation awareness despite extensive human factor research on cognition and everyday activities, such as driving and the use of new technologies (e.g., [46, 50]).

Measuring situation awareness

As important as developing effective training programs are to improving situation awareness, accurately assessing older adults' situation awareness is as important. Several methods have been used to assess situation awareness, including psychophysiological techniques, subjective techniques, performance measures, and questionnaires [17]. Researchers, however, need to understand the benefits and limitations of these before assessing one's level of situation awareness.

Psychophysiological techniques (e.g., EEG, P300, fMRI, eye tracking) examine the relationship between cognition and physiology by "manipulating" behaviors and then measuring physiology. For situation awareness, these techniques allow researchers to understand the perception and processing of environmental cues; however, the major limitation with this technique is in the ability to determine whether individuals are comprehending such cues, implementing the acquired information correctly, or retaining this information for future situations [17]. For these reasons, assessing situation awareness with psychophysiology should not be dismissed but rather combined with other objective and performance measures.

Subjective techniques, such as self-ratings and observer ratings, provide researchers with an easy, fast, inexpensive way of measuring situation awareness. Unfortunately, subjective techniques have significant limitations. Asking an individual to rate their own situation awareness following a task can be positively or negatively influenced by performance outcomes, and collecting self-ratings during a dynamic task takes away from an individuals' ability to perceive changes in the environment [17]. In addition, observer ratings cannot accurately assess a performer's concept of a situation regardless of how knowledgeable the observer is about the situation [17]. Overall, researchers should avoid subjective techniques when assessing situation awareness.

Performance measures can objectively assess situation awareness through computer-based simulations or during live situations. Required data, such as the speed and accuracy of responses, can be collected directly by computer programs or examined afterward through video-based analysis. Although performance measures produce the least amount of interference, there are disadvantages. Performance data are indirect measures of situation awareness and make it difficult to determine reasons for poor performance. Errors may have occurred in decision making, causing the performance to decrease and negatively reflect on situation awareness. By combining performance measures with direct assessments of situation awareness, greater knowledge can be attained on improvements in cognition and performance.

Finally, direct measurement of situation awareness comes in the form of questionnaires. Of all questionnaires used, the Situation Awareness Global Assessment Technique (SAGAT; [14]) was developed to assess each component of situation awareness (e.g., Levels 1, 2, and 3) and is the most widely used scale. The SAGAT is an objective tool that queries performers during simulated events. The SAGAT taps directly into the perceptions of dynamic performers rather than making post hoc inferences from behavioral observations or subjective self-ratings and provides an immediate, detailed understanding of situation awareness and the qualities that positively or negatively affect performance. The effectiveness of this technique lies in the development of queries, which can be created for any task-specific situation. A detailed explanation of the SAGAT as well as the abovementioned techniques goes beyond the scope of this paper; however, Endsley and Garland [20] provided a thorough description of each. With respect to aging, age-related declines may also influence the measurement of situation awareness. The next section will discuss how deficits explained by cognitive aging theories may contribute to age-related declines in situation awareness at each of the three levels.

\section{Age-related cognitive declines and situation awareness}

Four prominent theories have been proposed to explain the causes of age-related differences in cognitive functioning. These theories of cognitive aging include fluid versus crystallized intelligence [29, 47], inhibition deficits [27, 38], reduced processing resources [43, 53, 54], and cognitive slowing [6, 10, 53]. Empirical evidence in support of these theories paints a relatively dark picture for older adults showing increased age-related problems in many areas (e.g., the capacity to hold information in 
working memory, performing dual tasks simultaneously, and general slowing of responses as well as processing speed). How these cognitive declines might influence situation awareness is addressed below.

\section{Fluid versus crystallized intelligence}

Over the years, individuals acquire and store knowledge that is used regularly throughout life. Repetition causes this knowledge to develop into a meaningful database of information. The processes that access this accumulated knowledge of previous experiences are known as crystallized intelligence [29, 47]. For example, vocabulary is actively being acquired throughout early childhood and adolescence. Constant retrieval and use of vocabulary to communicate with others is a part of crystallized intelligence. In contrast, situations do occur where individuals are required to process comparatively new information. In these situations, the lack of previous experience and knowledge structures requires processes to tap into fluid intelligence. Researchers examining the effects of aging on fluid and crystallized intelligence have found declines in fluid intelligence; however, crystallized intelligence did not exhibit the same declines and often improved during these later years [29, 47].

During predictable and stable situations, crystallized intelligence remains constant, causing no detrimental effects on situation awareness. However, the importance of situation awareness lies in the benefits gained during unpredictable, dynamic environments. These situations call upon fluid intelligence to provide accurate comprehension of perceived cues. If the cues being presented were unfamiliar, causing fluid intelligence to successfully process new information, declines would occur in Level 1 situation awareness thus causing comprehension problems in Level 2. However, the necessary cues in a dynamic situation are often stable, leaving older adults' ability to comprehend the situation (i.e., Level 2 situation awareness) the sole determinant of a successful performance. Levels 2 and 3 situation awareness suffer the greatest deficits from declines in fluid intelligence, which is easily understood when one considers the required awareness necessary to drive in a new city. We have all driven behind a vehicle with out-of-state license plates and experienced the frustration of ill-timed turn signals, slow speeds, and overall erratic driving. Knowledge of driving as well as traffic laws, which are considered a part of crystallized intelligence, have not changed and are not difficult to call upon while driving on hometown streets. However, driving in an unfamiliar city during rush hour traffic elicits fluid intelligence to handle new information, and in new environments, deficits are likely to occur for older adults.
Processing resources

The amount and complexity of information entering the system, the difficulty of the task, and the relative diversity between dual tasks all compromise processing resources (e.g., processing speed, working memory, and attention) especially in older adults $[12,36,39,40,57,60]$. Aged individuals, suffering from attentional deficits, may ultimately have greater difficulties registering and encoding information efficiently. Declines in attentional operation may also have adverse effects on working memory, which in turn directly influence performance on tasks requiring situation awareness. Because dynamic situations and sports involve continuous perception and comprehension of information, processing resource deficits may cause the greatest decay in situation awareness relative to the other cognitive deficits mentioned.

Attentional resources are the single limiting factor during the perception of relevant cues (i.e., Level 1 situation awareness). Age-related declines directly affect the ability of older adults to selectively attend to information-rich cues in the environment, especially during situations where multiple stimuli are present. Moreover, divided attention further stresses perceptual abilities when dual tasks are being performed. Levels 2 and 3 situation awareness also suffer from age-related declines as the processing that occurs in these levels exists in working memory [7, 16]. Retrieving, classifying, and processing massive amounts of information may be complicated by inaccurate mental models created from Level 1, causing further declines in situation awareness. If a mental model did not exist for a four-way intersection and the driver approaching is unaware of the traffic with the right of way, weakened processing resources will heighten temporal constraints, possibly resulting in an accident. Similar situations could generate further problems when elderly drivers are unable to access previous situational information (e.g., driver is faced with another four-way intersection but in a different location).

\section{Inhibition deficits}

Hasher and Zacks [27] originally proposed that working memory would only operate efficiently when inhibitory mechanisms facilitated selective attention, limited irrelevant information, and allowed access to information that coincided with the "goal path." Because older adults have a more detailed knowledge base, specific situations may activate more information, causing interference in working memory [41]. For example, although Hashtroudi et al. [28] found that both younger and older adults encoded personal thoughts and feelings, only older adults were unable to inhibit these, which produced interference with retrieval 
processes and, more importantly, a reduction in perceptualbased memory.

Because perceptual-based memory is key to superior situation awareness, deficits in the ability to inhibit will cause deficits across all levels of situation awareness. During Level 1 of situation awareness, disinhibition causes immediate deterioration in selective attention. Subsequently, multiple environmental cues are accessed, making it difficult to limit irrelevant information. Moreover, these inhibition deficits tax working memory, causing further problems in Level 2 situation awareness. Once the correct cues have been perceived, accurately comprehending how these cues will influence the situation becomes bogged down in working memory. Disinhibition causes errors in the retrieval of information, which is directly tied to working memory and successful comprehension. Although inhibition does not directly influence Level 3 situation awareness, Level 3 will be impaired because of the deficits occurring in Levels 1 and 2. For example, inhibiting rules become more difficult when driving in a city such as Boston, where it is illegal to turn right on red, speed limits are distinctly slower than in rural areas, and traffic lights are located on the sidewalk as opposed to hanging over the middle of the street.

\section{Cognitive slowing}

Mental and physical operations gradually slow down during older adulthood [6, 53]. Slowing becomes a particular concern in highly dynamic situations as such situations are typically laden with temporal pressures. Salthouse [55] hypothesized that the limited time mechanism and the simultaneity mechanism are responsible for such drops in processing speeds. The limited time mechanism states that the time available for secondary operations is reduced because of the extended duration necessary to execute initial operations [55]. The simultaneity mechanism causes information from early processing to be lost or become obsolete by the time later processing is completed [55]. These processing declines directly relate to effective Levels 1 and 2 situation awareness.

First, consider the effects of the limited time mechanism on situation awareness. When the comprehension of cues (Level 2) is constrained because of the extensive processing required to perceive which cues are relevant (Level 1), the ability to comprehend these cues must either comprise of little processing effort or the perception of cues must be the determining factor of performance for successful outcomes to occur, which is not the case with situation awareness. In temporally driven, dynamic environments, the initial operation (i.e., Level 1) is equally important to the secondary operation (i.e., Level 2). Although an argument can be made for the importance of Level 1 situation awareness, effective situation awareness lies in the coordination of all three levels. Second, the effects of the simultaneity mechanism equally influence situation awareness because of the sequential type of processing required. When the information from Level 1 is lost because of extended processing of Level 2, appropriate mental models cannot be created. Furthermore, Level 1 will never be developed, weakening the cognitive benefits of situation awareness. A situation that accurately illustrates the effects of cognitive slowing on situation awareness, specifically with regards to the limited time and simultaneity mechanisms, can again be seen in many driving situations. When vital cues are presented regarding the speed of traffic and available lanes for entering an unanticipated construction area, loss of Level 1 information or constraining the time needed for Level 2 could cause life-threatening accidents.

\section{A model for training older adults' situation awareness}

Currently, Endsley [16] has proposed a comprehensive model of situation awareness, which outlines how elements from the environment as well as cognition influence situation awareness and decision making. When considering the beneficial effects of these processes, situation awareness may provide the key to combining these processes and improving the cognition in older adults. Figure 1 provides our expanded situation awareness model, which depicts these multidimensional connections and the cognitive declines associated with them. The core of the situation awareness model has been expanded to further depict the relationship between conceptual representation and situation awareness as well as theories of cognitive aging. This model is designed to stimulate future research on age-appropriate training of situation awareness, which has the potential to reduce cognitive declines and lead to an increased functioning in everyday life.

Baltes and Baltes' [2] theory of selective optimization with compensation proposes seven propositions for aging successfully, two of which directly relate to improvements that could be achieved from training situation awareness. Specifically, when considering the amount of latent reserve available in older adults, training situation awareness activates resources previously abandoned or negatively affected by new technology or changing environments. Through learning and practice, older adults may benefit from training to the same degree as younger adults [2]; however, reaching the same levels of performance outcomes (i.e., response speed and accuracy) as their younger counterparts may be unattainable. In addition, older adults' preexisting knowledge can offset losses that occur in their reserve capacity. Improving situation awareness, especially Level 3, allows older adults to utilize this past knowledge. 
Fig. 1 A model of situation awareness and its interactions with cognitive aging. The components of the model proposed by Endsley [16] are in nonitalicized text, whereas our added components are shown in italics

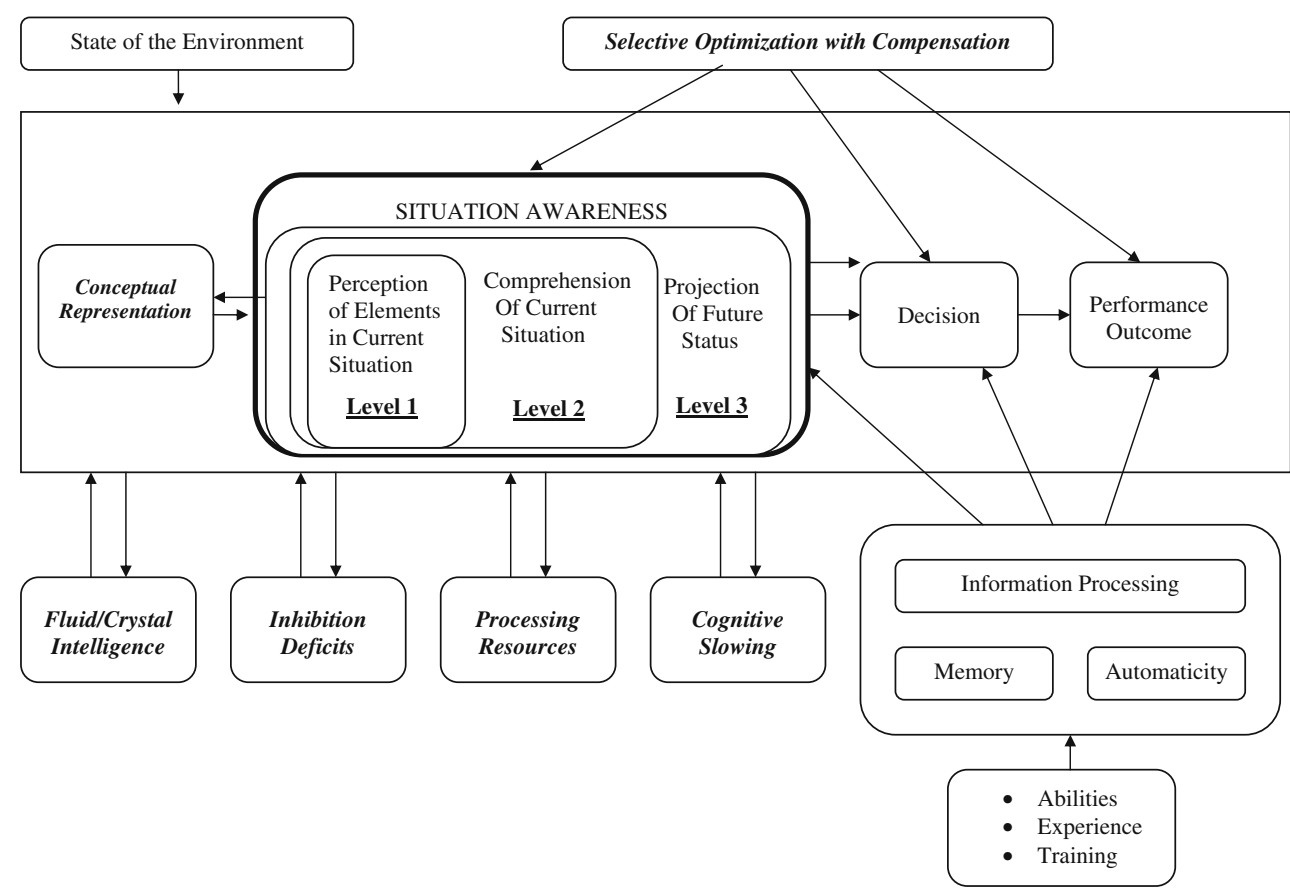

Recently, researchers examining the effects of cognitive training with aged individuals have found that interventions significantly improve specific cognitive abilities, including processing speed, reasoning, and memory (e.g., [1, 49]). In addition, the use of the Visual-Motor Useful Field of View training has been an effective tool for improving the psychomotor skills of senior drivers, which is most likely indirectly training situation awareness [32]. By directly training the core variable situation awareness (bold square), this key component permeates all other variables in the model. The following recommendations for training provide insight into the effects of training situation awareness and potential benefits on everyday task performance.

When considering each of the three levels of situation awareness, Level 1 can be easily trained with multiple methodologies. In any situation, regardless of how simple or complex the task, a small, specific set of cues provides the most relevant information concerning the potential outcome. For example, new kitchen stoves provide cues regarding which burners are still hot even after the stove has been turned off. Individuals only need to be directed to these relevant as opposed to irrelevant cues. Specifically, the use of video training as well as in situ training (i.e., training conducted in one's natural environment outside of laboratory settings) can be developed to explicitly or implicitly instruct older adults on relevant cues. Video training programs consist of real-time and slow motion clips that accurately and clearly depict the perceptual cues vital to enhancing situational awareness, whereas situ training allows individuals to actively perform during live situations. Based on the informational cues being highlighted during training, individuals can then begin to comprehend the outcome of interpreting those cues and decide more quickly and decisively an appropriate course of action. It is important to note that although sensory deficits (i.e., vision and hearing) naturally occur with aging, visual deficits generally do not negatively affect perception or limit the benefits in training situation awareness [4, 22]. Perception and situation awareness tap temporal-spatial abilities in which visual acuity, depth perception, and peripheral vision have no direct influence on performance outcomes. Declines in hearing, however, may negatively influence situation awareness when technological environments produce auditory signals that cue individuals to vital information.

The basis of situation awareness is to produce quick and accurate decisions during a variety of situations. Training Level 1 specifically addresses the attentional aspects of situation awareness as well as age-related differences in working memory. When only relevant cues are being processed, working memory executes cognitive operations more efficiently. Training Level 1 situation awareness may provide the greatest benefits for older adults' cognition. For example, irrelevant environmental details have been identified as one category of "off-goal-path" thoughts [27]. Improvements in attending to essential cues can reduce the number of "off-goal-path" ideas that enter working memory thus directly addressing problems with disinhibition. In particular, video-based training can pause clips at specified moments or black out areas on each clip to restrict the visual access to different perceptual fields. These techniques may produce the most significant improvements in inhibition because of the lack of extraneous information. 
Level 1 training may also be beneficial to declines in fluid intelligence. For the elderly, cooking or using a kitchen stove has in all actuality formed in crystallized intelligence; however, new technology may change appropriate perceptual cues and their locations. Problems may also occur in emergency situations where the elderly are required to make quick, accurate, and effective decisions, which tap into fluid intelligence. Serious injuries or declining performances may be reduced in situations that tax fluid intelligence if the elderly become actively aware of the use of advanced cue utilization and its influence on assessing the situation. Narrowing attentional focus to appropriate external cues will decrease errors in Level 1, which may otherwise occur in older adults suffering from deficits in fluid intelligence.

With the perception of essential cues enhancing Level 1 situation awareness, training must be extended to comprehending these cues (Level 2 situation awareness). To improve attention, processing, and working memory, older adults must learn how to effectively scan the environment. Faster response selection will reduce the temporal pressures that may exist in comprehension. Occluding or explicitly instructing older adults on appropriate cues (i.e., a red light on the stove) has little benefit if there is no understanding of what that means (i.e., the stove is still hot). By improving the comprehension of cues that may occur during unpredictable situations, older adults may then be able to make more accurate decisions from a seemingly infinite number of alternative responses. Specifically, the use of video-based training can provide a safe environment for examining the outcomes of effective and ineffective cue utilization, where in situ testing in some situations can be reasonably dangerous. By enhancing Level 2 situation awareness, accurate mental models are created when relevant cues are perceived, and appropriate leverage points become more apparent during predictable and unpredictable situations, which can limit the amount of danger that may exist in everyday activities.

When individuals have mastered Levels 1 and 2 situation awareness, their ability to project from past knowledge to current experiences is heightened (i.e., Level 3). However, training strategies that specifically enhance Level 3 situation awareness are more difficult to develop. Because Level 3 is based on past knowledge and defines expertise in dynamic situations, Level 3 situation awareness may not be trainable, only developing from years of deliberate practice and experience. Actively engaging the cognitive processing required for situation awareness to occur through videobased training of Levels 1 and 2 may initiate past experiences where Level 3 was necessary. The deliberate practice of Levels 1 and 2 will change situational processes from a conscious state to an unconscious, automatic process, which taps into Level 3. The attainment of Level 3 situation awareness and, more importantly, sustaining
Level 3, could potentially be the most influential factor in decreasing age-related effects on cognition as it relies on fewer resources and activates processes sooner.

To test the idea of training older adults on situation awareness, a recent study [9] used a multidimensional, perceptual-cognitive skills training protocol with senior tennis players who had no cognitive impairments $(n=13)$ or mild levels of cognitive impairment $(n=14)$. Participants included senior male $(n=10, M=62.5$ years old, $\mathrm{SD}=8.4)$ and female $(n=17, M=56.6, \mathrm{SD}=8.5)$ tennis players over the age of 50. Players competed regularly in the United States Tennis Association (USTA) league tournaments; thus, each had a National Tennis Rating Program (NTRP) certified rating. The average experience level of the participants, according to the NTRP, was $3.17(\mathrm{SD}=0.50)$, which categorizes them at the intermediate level. Before training, each participant was administered the Mini-Mental State Examination (MMSE; [21]) to test for cognitive impairments. Participants scoring between 20 and 24 points were considered to have mild levels of cognitive impairment, and those scoring 25 points or higher were considered noncognitively impaired. Participants were then individually pretested in a four-game singles match. Participants were randomly assigned to one of three groups as follows: (1) perceptual-cognitive skills training (PCST), (2) technique-footwork training (TFT), or (3) control. In the two training conditions, participants were provided with group-specific, standardized instructions on-court and with video to maximize the effects of training. Specifically, PCST consisted of developing the three levels of situation awareness, situational probabilities, player tendencies, anticipation, and decision making; whereas the TFT contained instructions regarding the improvement of groundstrokes, approach shots, volleys, overheads, serves, and return-of-serves as well as the corresponding footwork to assist efficiency and court coverage. The control group received no training. The PCST and TFT participants met on five consecutive days, whereas the control participants met only twice to complete pre-and posttesting.

Two experts on perceptual-cognitive skills, who also had extensive background in the game of tennis, examined relevant data from pre-and posttest video analysis. The two experts individually coded response speed, response accuracy, and performance decision making. Response speed was considered to be the time period from the point-ofcontact to the completion of the participants' first step in the correct direction of the ensuing shot. Response accuracy consisted of the percentage accuracy of on-court movement in relation to tracking toward the ensuing shot. Based on the accuracy of movement, participants were given one point for correctly moving in the appropriate direction and no points for moving in the wrong direction. Performance decision making was operationalized as the appropriateness 
of the participants' shot selections according to court positioning, percentages, situation, and shot hit by the opponent. Participants received one point for choosing appropriate performance decisions and no points for inappropriate performance decisions. Inter-rater reliability scores for the points to be examined, response speed, response accuracy, and performance decision making all exceeded $98 \%$.

The experimental design consisted of the three factors as follows: level of impairment (cognitive impairment, no impairment), training type (PCST, TFT, control), and test (pretest, posttest). The dependent measures were response speed, response accuracy, and decision making during singles match play situations. No significant differences were found between participants with cognitive impairments and those without cognitive impairments, as both groups benefited from PCST training. Findings indicated that noncognitively impaired participants who received PCST training (which included situation awareness) cut their response speeds nearly in half following training (pretest, 1,059 ms; posttest, $589 \mathrm{~ms}$ ), allowing movements toward the intended shot as the ball crossed their opponent's service line. This faster response occurred with enhanced response accuracy (pretest, $31 \%$; posttest, $88 \%$ ) as well. In addition, correct decisions more than doubled (pretest, $36 \%$; posttest, $81 \%$ ) during live on-court singles matches. Cognitively impaired senior tennis players who received PCST training showed as much benefit as noncognitively impaired players, responding more than twice as fast following training (pretest, $1,038 \mathrm{~ms}$; posttest, $534 \mathrm{~ms}$ ) and more than doubling their response accuracy (pretest: $32 \%$, posttest: $88 \%$ ) to shots hit during singles match situations. With regards to performancebased decisions, cognitively impaired participants also made twice as many appropriate decisions (pretest, $32 \%$; posttest, $81 \%$ ).

Participants receiving PCST training significantly improved response speed, response accuracy, and performance decision making from pre-to posttest when compared to the other two groups (TFT and control). On average, PCST participants improved their response speed by nearly a half second (pretest, 1,050 ms; posttest, $568 \mathrm{~ms}$ ), whereas TFT (pretest, 1,140 ms; posttest, 1,200 ms) and control (pretest, $1,250 \mathrm{~ms}$; posttest, $1,290 \mathrm{~ms}$ ) participants made no improvements. PCST faster responses occurred with enhanced response accuracy (pretest, 31\%; posttest, $88 \%$ ) as opposed to the TFT (pretest, 22\%; posttest, 21\%) and control (pretest, 17\%; posttest, 15\%) groups. In addition, senior tennis players provided with PCST more than doubled the number of correct decisions (pretest, 35\%; posttest, $81 \%$ ) during live on-court singles matches, where players receiving TFT (pretest, 30\%; posttest, 30\%) and no training (pretest, 25\%; posttest, 25\%) made no improvements.
With only $3.50 \mathrm{~h}$ of training, improvements in the perception of relevant cues (Level 1) and the comprehension of these cues regarding the outcome of a situation (Level 2) were attained. The ability for such individuals to attain Level 3 situation awareness would require continued training as well as longitudinal data to support the maintenance of these skills. In addition, significant improvements in performance decision making indicated that multidimensional perceptual-cognitive training improved the availability of processing resources and overall cognitive processing speed. However, the lack of retention tests limits the interpretation of these findings. Further research should consider determining whether these improvements are true learning effects or merely transient training effects as well as the duration of each training session and the most effective length of training.

These findings lend clear evidence that such skills are trainable in older adults and are not limited to those who have achieved elite levels of expertise in their domain of interest [42]. For the senior tennis players in this study, especially those experiencing cognitive declines, cognitive functioning was expanded to include acquisition of new knowledge $[3,7,8,56]$. If these improvements can occur in the dynamic situations that exist in singles tennis match play, valuable information for successful aging may transfer to everyday environments. Whether the training of perceptual-cognitive skills in dynamic sport domains can be implicitly learned in daily life situations (e.g., driving, falling incidents, obstacle avoidance, etc.) and still produce similar results, is an important consideration for future research. By cross-examining perceptual-cognitive skills in dynamic sport and life situations, a better understanding is gained regarding general or task-specific training. Although fitness training and cognitive training have enhanced cognitive vitality of older adults $[1,3,11,31,37,49]$, more research is needed on training dynamically based cognitive skills that enhance the cognitive functioning and performance of older adults in such environments.

Equally important to the improvements made by perceptual-cognitive training is the compounding effect physical activity has on cognitive functioning [37]. Colcombe and Kramer's [11] meta-analytic examination of fitness effects on older adults' cognitive functioning found that fitness training improved performance $(0.5 \mathrm{SD})$ on tests of executive processes, regardless of cognitive task, training program or duration, or participants' gender or age. In addition to executive processes, control processes and visuospatial processes also improved from increased physical activity, which are vital to and directly influence situation awareness. The combination of task-specific training of perceptualcognitive skills and fitness training may significantly impact the maintenance of active lifestyles, physical and cognitive health, and overall quality of life. 


\section{Conclusions}

In this review, we have examined situation awareness and its relevance to the cognitive declines experienced by older adults, and we have begun addressing the potential ameliorating effects of training situation awareness. By reformatting the prototypical representations of daily activities and technologically advanced products, older adults will be better prepared to selectively attend to changes in the environment. More importantly, updated conceptual representations allow for improved situation awareness. The perception of relevant cues (i.e., Level 1 situation awareness) and comprehension of those cues (i.e., Level 2 situation awareness) will improve responses to both predictable and unpredictable situations. Future research should examine how interventions based on situation awareness affect deficits in aging and improve performance in dynamic situations and physical activities. Considerations need to be made for the development of online training programs, which allow older adults access to perceptual-cognitive training in task-specific domains. Activities that become more dangerous as we age (e.g., obstacle avoidances, cooking, traveling) should be considered as well as those that maintain the quality of living (e.g., driving and sport activity).

\section{References}

1. Ball K, Berch DB, Helmers KF, Jobe JB, Leveck MD, Marsiske M, Morris JN, Rebok GW, Smith DM, Tennstedt SL, Unverzagt FW, Willis SL (2002) Effects of cognitive training interventions with older adults: a randomized controlled trial. JAMA 288:22712281

2. Baltes PB, Baltes MM (eds) (1990) Successful aging: perspectives from the behavioral sciences. Cambridge University Press, New York

3. Baltes PB, Lindenberger U (1988) On the range of cognitive plasticity in old age as a function of experience: 15 years of intervention research. Behav Ther 19:283-300

4. Baltes PB, Lindenberger U (1997) Emergence of a powerful connection between sensory and cognitive functions across the adult life span: a new window to the study of cognitive aging? Psychol Aging 12:12-21

5. Barsalou LW (2003) Situated simulation in the human conceptual system. Lang Cogn Process 18:513-562

6. Birren JE (1970) Toward an experimental psychology of aging. Am Psychol 25:124-135

7. Bolstad CA, Hess TM (2000) Situation awareness and aging. In: Endsley MR, Garland DJ (eds) Situation awareness analysis and measurement. Lawrence Erlbaum, Mahwah, NJ, pp 277-302

8. Bosman EA, Charness N (1996) Age related differences in skilled performance and skill acquisition. In: Blanchard-Fields F, Hess TM (eds) Perspectives on cognitive change in adulthood and aging. McGraw-Hill, New York, 428-453

9. Caserta RJ, Young J, Janelle C (2007) Old dogs, new tricks: training the perceptual skills of senior tennis players. J Sport Exercise Psychol (in press)
10. Cerella J (1994) Generalized slowing in brinley plots. J Gerontology Psychol Sci 49:P65-P71

11. Colcombe S, Kramer AF (2003) Fitness effects on the cognitive function of older adults: a meta-analytic study. Psychol Sci 14:125-130

12. Damos D, Wickens CD (1980) The acquisition and transfer of time-sharing skills. Acta Psychol 6:569-577

13. Drews FA, Westenskow DR (2006) The right picture is worth a thousand numbers: data displays in anesthesia. Hum Factors 48:59-71

14. Endsley MR (1987) SAGAT: a methodology for the measurement of situation awareness (NOR DOC 87-83). Northrop, Hawthorne, CA

15. Endsley MR (1988) Design and evaluation for situation awareness enhancement. In: Proceedings of the Human Factors Society 32nd Annual Meeting. Human Factors Society, Santa Monica, CA, pp 97-101

16. Endsley MR (1995) Towards a theory of situation awareness. Hum Factors 37:32-64

17. Endsley MR (1995) Measurement of situation awareness in dynamic systems. Hum Factors 37:65-84

18. Endsley MR (2000) Theoretical underpinnings of situation awareness: a critical review. In: Endsley MR, Garland DJ (eds) Situation awareness analysis and measurement. Lawrence Erlbaum, Mahwah, NJ, pp 3-32

19. Endsley MR, Bolstad CA (1994) Individual differences in pilot situation awareness. Int J Aviat Psychol 4:241-264

20. Endsley MR, Garland DJ (eds) (2000) Situation awareness analysis and measurement. Lawrence Erlbaum, Mahwah, NJ

21. Folstein MF, Folstein SE, McHugh PR (1975) Mini-mental state: a practical method for grading the cognitive state of patients for the clinician. J Psychiatr Res 12:189-198

22. Fozard JL (1990) Vision and hearing in aging. In: Birren JE, Schaie KW (eds) Handbook of the psychology of aging. Academic, New York, pp 150-171

23. Gaba DM, Howard SK, Small SD (1995) Situation awareness in anesthesiology. Hum Factors 37:20-31

24. Gales BJ, Menard SM (1995) Relationship between the administration of selected medications and falls in hospitalized elderly patients. Ann Pharmacother 29:354-358

25. Hampton JA, Ross HE (2003) Concepts and meaning: introduction to the special issue on conceptual representation. Lan Cogn Process 18:505-512

26. Hasher L, Stolzfus ER, Zacks RT, Rypma B (1991) Age and inhibition. J Exp Psychol Learn Mem Cogn 17:163-169

27. Hasher L, Zacks RT (1988) Working memory, comprehension, and aging: a review and a new view. In: Bower GH (ed) The psychology of learning and motivation. Academic, San Diego, CA, pp 193-225

28. Hashtroudi S, Johnson MK, Chrosniak LD (1990) Aging and qualitative characteristics of memories for perceived and imagined complex events. Psychol Aging 5:119-126

29. Horn JL, Cattell RB (1966) Age differences in primary mental ability factors. J Gerontology 21:210-220

30. Jones DG, Endsley MR (1996) Sources of situation awareness error in aviation. Aviat Space Environ Med 67:507-512

31. Jones S, Nyberg L, Sandblom J, Neely AS, Ingvar M, Petersson KM, Backman L (2006) Cognitive and neural plasticity in aging: general and task-specific limitations. Neurosci Biobehav Rev 30:864-871

32. Klavora P, Heslegrave RJ (2002) Senior drivers: an overview of problems and intervention strategies. J Aging Phys Activ 10:322335

33. Klein GA (1989) Recognition-primed decisions. In: Rouse WB (ed) Advances in man-machine systems research. JAI, Greenwich, CT, pp 47-92

34. Klein GA, Crandall BW (1995) The role of mental simulation in naturalistic decision-making. In: Hancock P, Flach J, Caird J, 
Viente K (eds) Local applications of the ecological approach to human-machine systems. Lawrence Erlbaum, Hillsdale, NJ, pp 324-358

35. Klein GA, Wolf S (1998) The role of leverage points in option generation. IEEE Trans Sys Man Cybernet 28:157-160

36. Korteling JE (1993) Effects of age and task similarity on dual-task performance. Hum Factors 35:99-114

37. Kramer AF, Hahn S, Cohen NJ, Banich MT, McAuley E, Harrison CR, Chason J, Vakil E, Bardell L, Boileau RA, Colcombe A (1999) Ageing, fitness and neurocognitive function. Nature 400:418-419

38. Layton B (1975) Perceptual noise and aging. Psychol Bull $82: 875-883$

39. Lorsbach TC, Simpson GB (1988) Dual-task performance as a function of adult age and task complexity. Psychol Aging 3:210212

40. McDowd JM, Craik FIM (1988) Effects of aging and task difficulty on divided attention performance. J Exp Psychol Hum Percept Perform 14:267-280

41. McDowd JM, Oseas-Kreger DM, Filion DL (1995) Inhibitory processes in cognition and aging. In: Dempster FN, Brainerd CJ (eds) Interference and inhibition in cognition. Academic, San Diego, CA, pp 363-400

42. Morrow D, Leirer V, Altieri P, Fitzsimmons C (1994) When expertise reduces age differences in performance. Psychol Aging 9:134-148

43. Navon D (1984) Resources - a theoretical soupstone? Psychol Rev 91:216-234

44. O'Hare D (1997) Cognitive ability determinants of elite pilot performance. Hum Factors 39:540-552

45. Olson D (1970) Language and thought: aspects of a cognitive theory of semantics. Psychol Rev 77:257-273

46. Parasuraman R, Nestor PG (1991) Attention and driving skills in aging and alzheimer's disease. Hum Factors 33:539-557

47. Rabbitt PMA (1993) Crystal quest: a search for the basis of maintenance of practiced skills into old age. In: Baddeley A, Weiskrantz L (eds) Attention: selection, awareness, and control. Clarendon, Oxford, UK, pp 188-230

48. Rodgers MD, Mogford RH, Strauch B (2000) Post hoc assessment of situation awareness in air traffic control incidents and major aircraft accidents. In: Endsley MR, Garland DJ (eds) Situation awareness analysis and measurement. Lawrence Erlbaum, Mahwah, NJ, pp 73112

49. Roenker DL, Cissell GM, Ball K, Edwards JD (2003) Speed-ofprocessing and driving simulator training result in improved driving performance. Hum Factors 45:218-233

50. Rogers WA, Fisk AD, Mead SE, Walker N, Cabrera EE (1996) A survey of automatic teller machine usage across the adult lifespan. Hum Factors 38:425-433

51. Regal DM, Rogers WH, Boucek G (1988) Situation awareness in the commercial flight deck: definition, measurement, and enhancement. In: Proceedings of the Seventh Aerospace Behavioral Technology Conference and Exposition. Society of Automotive Engineers, Warrendale, PA, pp 65-69

52. Rowe MA, Fehrenbach N (2004) Injuries sustained by community-dwelling individuals with dementia. Clin Nurs Res 13:98110

53. Salthouse TA (1985) Speed of behavior and its implication for cognition. In: Birren JE, Schaie KW (eds) Handbook of the psychology of aging. Reinhold, New York, pp 400-426

54. Salthouse TA (1988) Initiating the formalization of theories of cognitive aging. Psychol Aging 3:3-16

55. Salthouse TA (1996) The processing-speed theory of adult age differences in cognition. Psychol Rev 103:403-428

56. Schaie KW, Willis SL (1986) Can adult intellectual decline be reversed? Dev Psychol 22:223-232

57. Schneider W, Fisk AD (1982) Concurrent automatic and controlled visual search: can processing occur without costs? J Exp Psychol Learn Mem Cogn 8:261-278

58. Schyns PG, Goldstone RL, Thibaut JP (1998) The development of features in object concepts. Behav Brain Sci 21:1-54

59. Tinetti ME, Inouye SK, Gill TM, Doucette JT (1995) Shared risk factors for falls, incontinence, and functional dependence. Unifying the approach to geriatric syndromes. JAMA 273:1348-1353

60. Tun PA, Wingfield A (1997) Language and communication. In: Fisk AD, Rogers WA (eds) Handbook of human factors and the older adults. Academic, San Diego, CA, pp 125-149

61. Weerdesteyn V, Rijken H, Geurts ACH (2006) A five-week exercise program can reduce falls and improve obstacle avoidance in the elderly. Gerontology 52:131-141 